\title{
Light-controlled out-of-equilibrium assembly of cyclodextrins in an enzyme-mediated dynamic system
}

\author{
Larsen, Dennis; Bjerre, Philip M.; Beeren, Sophie R.
}

Published in:

Chemical Communications

Link to article, DOI:

$10.1039 / \mathrm{c} 9 \mathrm{cc} 08452 \mathrm{e}$

Publication date:

2019

Document Version

Peer reviewed version

Link back to DTU Orbit

Citation (APA):

Larsen, D., Bjerre, P. M., \& Beeren, S. R. (2019). Light-controlled out-of-equilibrium assembly of cyclodextrins in an enzyme-mediated dynamic system. Chemical Communications, 55(100), 15037-15040.

https://doi.org/10.1039/c9cc08452e

\section{General rights}

Copyright and moral rights for the publications made accessible in the public portal are retained by the authors and/or other copyright owners and it is a condition of accessing publications that users recognise and abide by the legal requirements associated with these rights.

- Users may download and print one copy of any publication from the public portal for the purpose of private study or research.

- You may not further distribute the material or use it for any profit-making activity or commercial gain

- You may freely distribute the URL identifying the publication in the public portal

If you believe that this document breaches copyright please contact us providing details, and we will remove access to the work immediately and investigate your claim 


\section{Light-controlled out-of-equilibrium assembly of cyclodextrins in an enzyme-mediated dynamic system}

Received 00th January 20xx,

Dennis Larsen, Philip M. Bjerre and Sophie R. Beeren*

Accepted 00th January 20xx

DOI: $10.1039 / x 0 x \times 00000 x$

We show that the selective enzymatic synthesis of specific cyclodextrins can be modulated using light. We use enzymemediated dynamic combinatorial chemistry to generate a mixture of interconverting linear and cyclic $\alpha$-1,4-glucans, and employ an azobenzene photoswitch as a template. Using UV or blue light to switch between photostationary states with different azobenzene cis/trans isomeric ratios, we can promote the out-of-equilibrium assembly of either $\alpha$-cyclodextrin or $\beta$-cyclodextrin.

Light-responsive systems in living organisms exploit photoswitchable co-factors to trigger the onset of enzymecatalysed reaction cascades to modulate growth and control essential functions. For example, phototropic plants optimize their growth in response to sunlight via the judicious synthesis of specific poly- and oligosaccharides, ${ }^{1}$ while the cis-trans photoisomerisation of retinal is responsible for visual transduction. ${ }^{2}$ Inspired by Nature, chemists have developed artificial light-responsive systems wherein a photo-activated conformation change in a small molecule or molecular moiety leads to a specific output from a chemical network, or altered physical properties of a self-assembled material. ${ }^{3}$

A number of artificial systems have recently been reported in which enzyme activity is controlled using light, either by direct bioconjugation of enzymes with photo-responsive moieties, or by photoisomerisation-based activation of a cofactor or deactivation of an inhibitor. ${ }^{4}$ More complex systems use light-controlled self-assembly of supramolecular structures for compartmentalization or release of co-factors or substrates, thus stimulating enzyme activity. ${ }^{5}$ Here we apply a unique strategy to control the outcome of an enzymatic process using light, which relies upon the use of a photo-responsive template to select different products in an enzyme-mediated dynamic system. Specifically, we control the preferential enzyme-

Department of Chemistry, Technical University of Denmark, Kemitorvet 207, DK2800 Kongens Lyngby, Denmark.

Electronic Supplementary Information (ESI) available: Experimental details, full list of photoswitches screened, chromatographic data, UV-vis spectra, NMR spectra. See DOI: $10.1039 / x 0 x \times 00000 x$ catalysed synthesis of $\alpha$ - or $\beta$-cyclodextrin via the photoisomerisation of an azobenzene template (Fig. 1).

Dynamic combinatorial chemistry is a now well-established
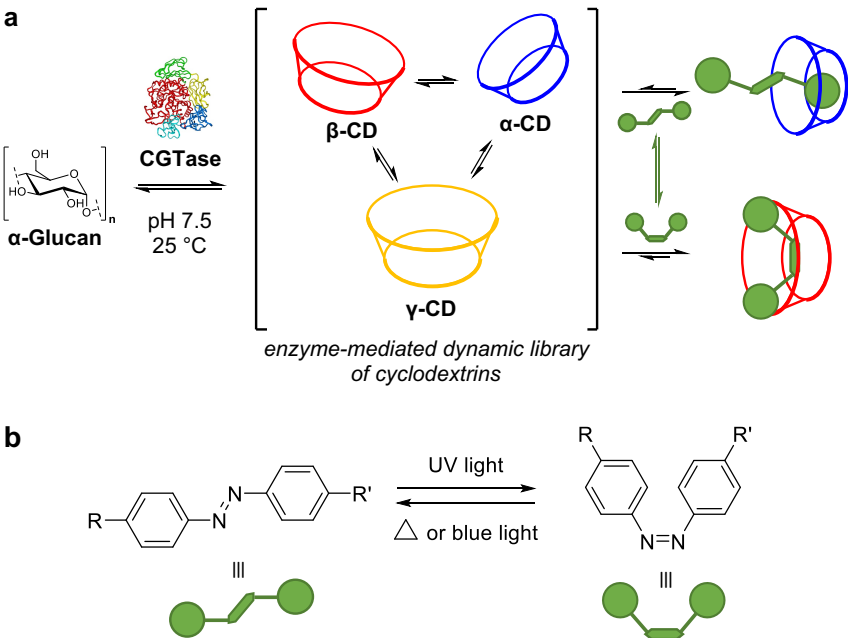

Figure 1 Light-controlled selective, enzyme-mediated synthesis of cyclodextrins. (a) CGTase acts on an $\alpha-1,4$-glucan source to generate a dynamic combinatorial library of cyclodextrins. Different isomers of the azobenzene template promote the selective synthesis of different cyclodextrins. (b) Azobenzenes switch from the trans- to the cisisomer upon irradiation with UV light and switch back thermally or with blue light.

methodology for the templated synthesis of complex molecular architectures, and the assembly of dynamic polymers and responsive systems from small building blocks under thermodynamic control. ${ }^{6}$ A few examples of dynamic combinatorial libraries based on reversible organic reactions (e.g. disulfide and hydrazone exchange) that incorporate lightresponsive building blocks ${ }^{7}$ and templates ${ }^{8}$ have been reported.

We recently described enzyme-mediated dynamic combinatorial chemistry, wherein enzyme-catalysed reactions are used to reversibly link molecular building blocks and generate mixtures of interconverting bio-oligomers. ${ }^{9}$ We employed cyclodextrin glucanotransferase (CGTase) $)^{10}$ to generate a dynamic mixture of linear $\alpha$-1,4-glucans 
(maltooligosaccharides) and cyclic $\alpha$-1,4-glucans (cyclodextrins (CDs)). We outlined how CGTase catalyses the fast, reversible transglycosylation and slow, irreversible hydrolysis of $\alpha(1 \rightarrow 4)$ glycosidic linkages, and thus generates a complex dynamic chemical system, wherein cyclodextrins assemble transiently out-of-equilibrium in a kinetically trapped subsystem. This subsystem operates under pseudo-thermodynamic control and by adding different templates, we were able to alter the distribution of the different cyclodextrins formed to obtain either $\alpha-C D, \beta-C D$ or $\gamma-C D$, with high selectivities ranging from $89 \%$ to $99 \%{ }^{9}$

Azobenzene moieties have been extensively utilised to achieve photo-responsive behaviour in functional molecular and biomolecular systems. ${ }^{11}$ Irradiation with UV light (typically 340-380 nm) causes a photo-induced isomerisation from the most stable trans isomer to the more sterically encumbered and less stable cis isomer (Fig. 1b). ${ }^{12}$ Return to the trans isomer occurs via a thermal back-reaction, or can be promoted by irradiation with visible light (typically 430-550 nm). Sparsely substituted azobenzenes are known to bind within the hydrophobic cavities of cyclodextrins (in aqueous solution) and the selective recognition of the cis or trans isomers has been exploited to control self-assembly of functional materials. ${ }^{13}$ For this study, we screened a number of different substituted azobenzenes (ESI Table S1) and identified a set of water soluble azobenzene photoswitches (1-5) exhibiting markedly different cis/trans isomeric ratios in ambient light and when irradiated with UV light at $365 \mathrm{~nm}$ (Table 1 and ESI Figs S2 and S3).

To investigate the possibility of modulating the selective synthesis of different cyclodextrins using light, we examined the action of CGTase on $\alpha-C D(2 \mathrm{mg} / \mathrm{ml}$ in $50 \mathrm{mM}$ sodium phosphate buffer at $\mathrm{pH} 7.5)$ in the presence of azobenzenes 1-5 (2 mM), in the dark, or with UV-irradiation ( $365 \mathrm{~nm}$, for 1 hour prior to addition of enzyme and continuously throughout the reaction). The composition of each library was analysed after 2 hours, using high performance liquid chromatography with evaporative light-scattering detection (HPLC-ELS), and compared with an untemplated reaction (Fig. 2 and ESI Fig. S4).

Table 1 Physicochemical properties of azobenzenes $\mathbf{1}-\mathbf{5}^{a}$

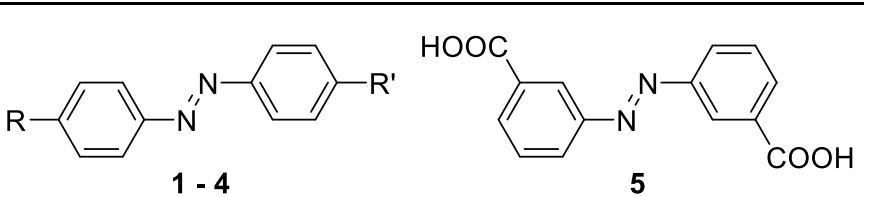

\begin{tabular}{ccccccc}
\hline & \multicolumn{2}{c}{ Photoswitch } & \multicolumn{2}{c}{$\lambda_{\max }(\mathrm{nm})$} & \multicolumn{2}{c}{${\text { cis/trans } \text { ratio }^{b}}^{-}$} \\
\hline & $\mathrm{R}$ & $\mathrm{R}^{\prime}$ & trans & cis & No irr & UV \\
\hline $\mathbf{1}$ & $\mathrm{COOH}$ & $\mathrm{H}$ & 326 & 426 & $15: 85$ & $73: 27$ \\
$\mathbf{2}$ & $\mathrm{COOH}$ & $\mathrm{COOH}$ & 331 & 429 & $10: 90$ & $51: 49$ \\
$\mathbf{3}$ & $\mathrm{SO}_{3} \mathrm{Na}$ & $\mathrm{OAC}$ & 325 & 426 & $12: 88$ & $63: 27$ \\
$\mathbf{4}$ & $\mathrm{SO}_{3} \mathrm{Na}$ & $\mathrm{NHAC}$ & 354 & 420 & $1: 99$ & $74: 26$ \\
$\mathbf{5}$ & & & 320 & 423 & $18: 82$ & $70: 30$ \\
\hline
\end{tabular}

${ }^{a}$ At room temperature in $50 \mathrm{mM}$ sodium phosphate buffer at $\mathrm{pH} 7.5 .{ }^{b}$ By ${ }^{1} \mathrm{H}$ NMR spectroscopy when kept in ambient light (no irr.) or after irradiation with light (365 $\mathrm{nm}$ ) for 1 hour (UV).

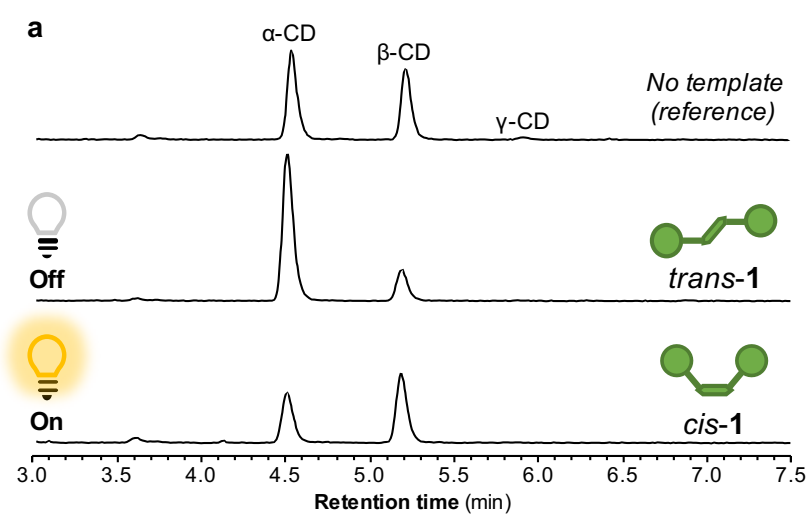

b

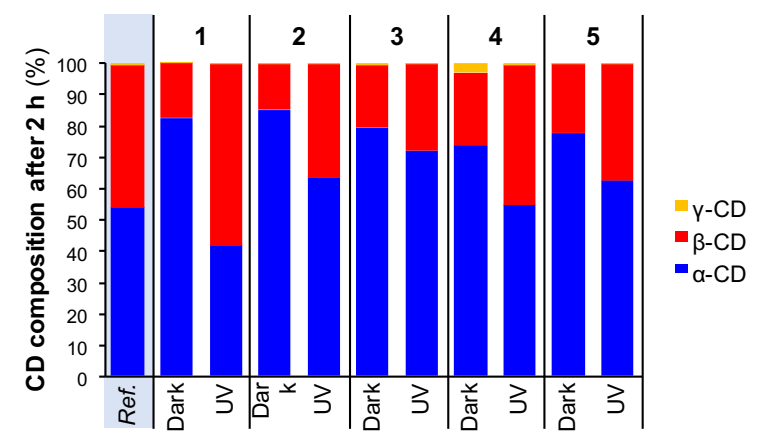

Figure 2 Screening of azobenzenes 1-5 as light-responsive templates for preferential synthesis of different cyclodextrins in a CGTase-mediated dynamic combinatorial library. (a) Representative chromatograms (HPLC-ELS) depicting the relative concentrations of $\alpha-, \beta-$, and $\gamma-C D$ formed when CGTase acts on $\alpha-C D$ in the absence of template, and with $1(2 \mathrm{mM})$ in the dark or under UV-irradiation (365 nm). (b) Bar graph depicting the cyclodextrin distributions produced in similar reactions templated with azobenzenes $1-$ 5. Reaction time: 2 hours. Conditions: $2 \mathrm{mg} / \mathrm{mL} \alpha$-CD used as starting $\alpha$-glucan in $50 \mathrm{mM}$ sodium phosphate buffer at $\mathrm{pH} 7.5$.

Pleasingly, in all cases, we observed different distributions of cyclodextrin products depending on whether or not the reaction was irradiated with UV-light. HPLC chromatograms showing the differing distributions of cyclodextrins that are formed in the absence of any template and in the presence of photoswitch 1, in the dark and with UV irradiation, are shown in Fig. $2 a$. In each of these reactions, $\alpha-C D$ and $\beta-C D$ were the major products (very little $\gamma-C D$ is produced at low concentrations of $\alpha-1,4$-glucan $(2 \mathrm{mg} / \mathrm{mL})$ ). A clear increase in the production of $\alpha-C D$ and concurrent decrease in the production of $\beta-C D$ was apparent upon addition of template 1 in the dark. Conversely, an increase in the production of $\beta-C D$ was observed upon application of UV-light together with the template. In the absence of template, UV-irradiation did not influence the distribution of products.

For each of the azobenzenes $\mathbf{1 - 5}$, addition of the template without irradiation generated higher concentrations of $\alpha-C D$, indicating that in each case the trans isomer binds preferentially to $\alpha$-CD (Fig. 2b). For photoswitches $\mathbf{2}-\mathbf{5}$, irradiation led to a reduction in the amplification of $\alpha-C D$ suggesting that either the cis-isomers have little interaction with either of the cyclodextrins, or there is a favourable interaction between the cis-isomers and $\beta-C D$, but this is outweighed by the interaction between $\alpha-C D$ and any remaining trans-isomer (note that the 

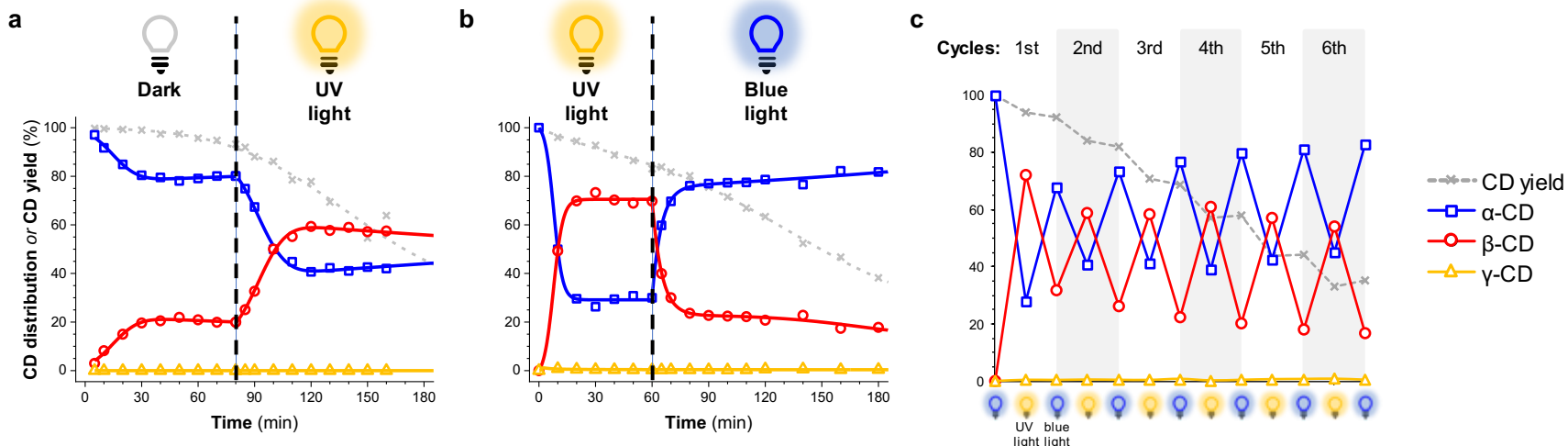

Figure 3. Light-controlled changes in CD distribution in CGTase-mediated dynamic cyclodextrin systems generated starting from $\alpha$-CD (2 mg/mL) and templated with photoswitch 1 (2 mM). a) Dynamic system kept in the dark before exposure to UV light ( $365 \mathrm{~nm}$ ). b) Dynamic system started under UV light irradiation (365 nm), then irradiated with blue light $(460 \mathrm{~nm})$. c) Consecutive cycles of irradiation with UV light ( $365 \mathrm{~nm}, 20$ minutes) and blue light (460 nm, 15 minutes).

photostationary state obtained under UV irradiation contains significant amounts of the trans isomers for all of the azobenzene templates, Table 1 ). Only photoswitch 1 caused the amplification of $\beta-C D$ when irradiated, indicating that cis-1 binds preferentially to $\beta-C D$, while trans-1 binds more strongly to $\alpha-C D$.

Determination of association constants for the binding of a guest that exists as a mixture of slowly interconverting isomers is not straightforward, as the isomeric ratio is altered by selective interaction with the host. As photoisomerization of azobenzenes does not give complete trans to cis conversion, the cis isomer is always contaminated with a minority of the trans isomer even after photoirradiation. This means that simple titrations, and fitting to a 1-to-1 binding model, cannot be used to accurately determine association constants for binding of cisisomers, due to competitive binding by the trans isomer minority. We have previously described how an NMR titration can be used to simultaneously determine association constants for the binding of a host to a number of different guests present in a mixture. ${ }^{14}$ This methodology does not require the concentrations of the host and guests to be known, nor should one be kept constant. It requires only that the association constant $\left(K_{\mathrm{a}}\right)$ is known for one of the guests. Here we show that the same methodology can be used to determine the association constants for cis-1 and trans-1 binding to $\alpha-C D$ and $\beta$-CD. Solutions of photoswitch 1 ( $1 \mathrm{mM}$ in $50 \mathrm{mM}$ sodium phosphate buffer at $\mathrm{pH} 7.5$ ) were titrated with $\alpha-C D$ and $\beta-C D$, with each titration performed under two different sets of conditions: (1) in the dark and after heating at $80^{\circ} \mathrm{C}$ for 2 days to obtain exclusively trans-1; (2) in ambient light, such that a mixture of cis-1 and trans-1 were present. For the titrations performed in the dark, association constants, $K_{\mathrm{a}(\text { trans) }}$ (Table 2 ) were obtained by fitting to a regular 1:1 binding isotherm (ESI Fig. S4). For the titrations in ambient light, both cis-1 and trans1 were present, and these interacted unequally with the cyclodextrins, leading to a change in the ratio of isomers present upon increasing the concentration of cyclodextrin. By plotting the changes in chemical shift observed for each isomer upon addition of cyclodextrin against one another $\left(\Delta \delta_{\text {cis }}\right.$ vs. $\left.\Delta \delta_{\text {trans }}\right)$, and fitting to the equation below, we could obtain $K_{\mathrm{a}(\mathrm{c} i \mathrm{~s})}$ for each cyclodextrin (Table 2 and ESI Fig. S5).

$\Delta \delta_{\text {cis }}=\frac{\Delta \delta_{\max (\text { cis })} \Delta \delta_{\text {trans }} K_{a(\text { cis })}}{\Delta \delta_{\max (\text { trans })} K_{a(\text { trans })}-\Delta \delta_{\text {trans }} K_{a(\text { trans })}+\Delta \delta_{\text {trans }} K_{a(\text { cis })}}$

Table 2. Association constants, $K_{\mathrm{a}}\left(\mathrm{M}^{-1}\right)$, for $\alpha$ - and $\beta-\mathrm{CD}$ with trans and cis isomers of $\mathbf{1}^{a}$

\begin{tabular}{ccc} 
& \multicolumn{3}{c}{ Guest } \\
Host & trans-1 & cis-1 \\
\hline$\alpha-C D$ & $5000 \pm 400$ & $140 \pm 70$ \\
$\beta-C D$ & $2500 \pm 300$ & $2200 \pm 300$ \\
\hline
\end{tabular}

$a$ In sodium phosphate buffer $(\mathrm{pH} 7.5,50 \mathrm{mM})$ in $\mathrm{D}_{2} \mathrm{O}$ at $25^{\circ} \mathrm{C}$.

The relative affinities of cis-1 and trans-1 for $\alpha-C D$ and $\beta-C D$ can thus explain the different product distributions obtained when CGTase catalyses the formation of CDs in the absence or presence of UV irradiation. When the enzymatic reaction is carried out in the dark, trans-1 predominates, and since it binds $\alpha-C D$ approximately twice as strongly as $\beta-C D$, the production of $\alpha-C D$ is amplified. When exposed to UV light, cis-1 predominates, and higher production of $\beta-C D$ results, because cis-1 binds 15 times more strongly to $\beta-C D$ than to $\alpha-C D$.

To illustrate how the dynamic enzymatic synthesis of cyclodextrins can be coupled to the dynamic switching between photostationary states of photoswitch $\mathbf{1}$, the CGTase-catalysed interconversion of CDs was monitored over time under varying light conditions. Firstly, a reaction was started in the dark and kept in darkness for the first 80 minutes before being submitted to UV-irradiation ( $365 \mathrm{~nm}$ ) (Fig. 3a). The system adapted quickly to the UV light, switching readily from 80:20 $\alpha-C D / \beta-C D$ ratio in the dark to the expected 40:60 ratio when under UV irradiation. A second reaction was begun under UV irradiation, and after 60 minutes, the irradiation was switched to blue light $(460 \mathrm{~nm})$ (Fig 3b). Irradiation with blue light returns the mainly cis-1 to trans1 , and thus the $\alpha-C D / \beta-C D$ ratio returned to approximately $80: 20$. Consecutive cycles of irradiation with UV-light (365 nm, 20 mins) and blue light ( $460 \mathrm{~nm}, 15 \mathrm{mins}$ ) were performed, demonstrating the possibility to oscillate up to six times between CD mixtures of predominantly $\alpha-C D$ or $\beta-C D$ (Fig 3c). 
Due to the irreversible background hydrolysis of glycosidic linkages, and the gradual accumulation of glucose (which cannot act as a glycosyl donor in CGTase-mediated glycosyl transfer), a steady decrease in the total cyclodextrin concentration is observed (Fig. 3 grey line and ESI Fig. S6-9). This explains the gradual rise in the $\alpha-C D$ concentration and concomitant descent in the $\beta-C D$ concentration, as at lower building block concentrations, shorter oligomers are favoured in dynamic combinatorial libraries. ${ }^{15}$ Background hydrolysis appears faster during UV-irradiation but this is presumably due to the overall weaker binding by both cyclodextrins to cis-1 compared to trans-1, and as we have previously shown, hydrolysis of cyclodextrins is hindered by complexation. ${ }^{9}$

In our enzyme-mediated dynamic system, cyclodextrins are not formed under equilibrium conditions, but instead selfassemble transiently out-of-equilibrium and are slowly converted to glucose, the thermodynamic product. ${ }^{9}$ Surprisingly, templates can nevertheless be exploited to select for specific cyclodextrin products, presumably because $\alpha-, \beta-$, and $\gamma-C D$ are separated only by low energy barriers and form a kinetically-trapped subsystem operating under pseudothermodynamic control. This azobenzene-templated system is doubly dynamic, as the equilibria for both the cis/trans isomerization and the CGTase-catalysed $\alpha-C D / \beta-C D$ interconversion influence one another. The unconventional energy landscape for this synthetic dynamic system is depicted in Figure 4. Here we show that we can use UV light to push the system further out-of-equilibrium and transiently occupy an alternative, higher energy dissipative, but also kinetically trapped state. ${ }^{16}$ Using different wavelengths of light to switch between predominantly trans-1 or cis-1, we can control which kinetically trapped regime is occupied, and promote the selective formation of $\alpha-C D$ or $\beta-C D$, respectively.

\section{Conclusions}

In conclusion, we have demonstrated the use of light to artificially control an enzyme-driven process, not by activation of a substrate or co-factor, but by exploiting the molecular recognition of a light-responsive template to select specific products in an enzyme-mediated dynamic system.

\section{Conflicts of interest}

There are no conflicts to declare.

\section{Notes and references}

1 E. Liscum, S. K. Askinosie, D. L. Leuchtmann, J. Morrow, K. T. Willenburg and D. R. Coats, Plant Cell, 2015, 26, 38-55.

2 K. Palczewski, J. Biol. Chem., 2012, 287, 1612-1619.

3 (a) Z. L. Pianowski, Chem. Eur. J., 2019, 25, 5128-5144; (b) A. K. Mandal, M. Gangopadhyay and A. Das, Chem. Soc. Rev., 2015, 44, 663-676; (c) J.-F. Gohy and Y. Zhao, Chem. Soc. Rev., 2013, 42, 7117-7129; (d) C. Zhu, C. Ninh and C. J. Bettinger, Biomacromol., 2014, 15, 3474-3494.

4 (a) T. Tian, Y. Song, J. Wang, B. Fu, Z. He, X. Xu, A. Li, X. Zhou, S. Wang and X. Zhou, J. Am. Chem. Soc., 2016, 138, 995-961;

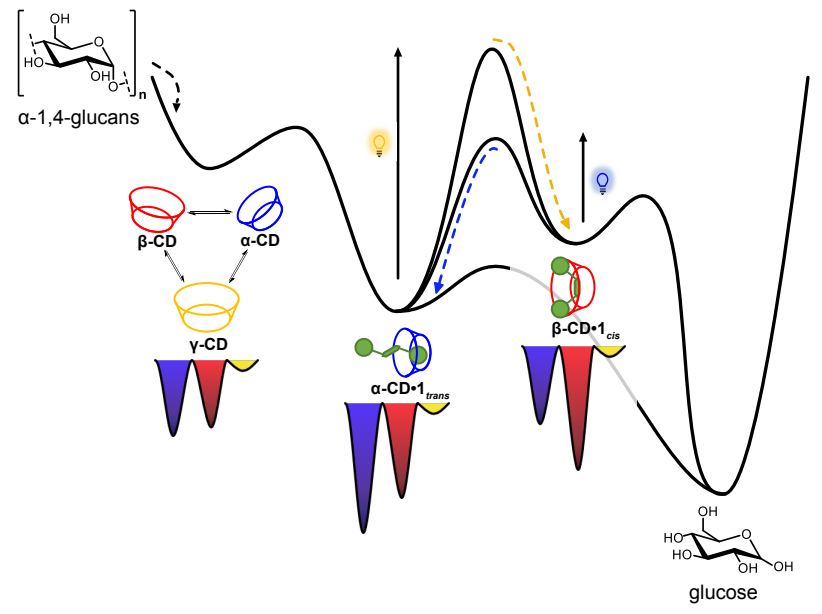

Figure 4 Schematic energy diagram summarizing the different energy levels populated in this photodynamic enzyme-mediated dynamic system. Photoactivation with UV or blue light shifts the cyclodextrin subsystem from one kinetically trapped minimum to another.

(b) A. Ali, G. A. Bullen, B. Cross, T. R. Dafforn, H. A. Little, J. Manchester, A. F. A. Peacock and J. H. R. Tucker, Chem. Commun., 2019, 55, 5627-5630; (c) K. Aggarwal, M. Banik, B. Medellin and E. L. Que, Biochemistry, 2019, 58, 48-53; (d) R. Mogaki, K. Okuro and T. Aida, J. Am. Chem. Soc., 2017, 139, 10072-10078; (e) W. Szymański, J. M. Beierle, H. A. V. Kistemaker, W. A. Velema and B. L. Feringa, Chem. Rev., 2013, 8, 6114-6178.

5 (a) S. Neri, S. G. Martin, C. Pezzato and L. Prins, J. Am. Chem. Soc., 2017, 139, 1794-1797; (b) J. W. Hindley, Y. Elani, C. M. McGilvery, S. Ali, C. L. Bevan, R. V. Law and O. Ces, Nat. Comm., 2018, 9:1093.

6 (a) P. Frei, R. Hevey and B. Ernst, Chem. Eur. J., 2019, 25, 6173; (b) F. B. L. Cougnon and J. K. M. Sanders, Acc. Chem. Res., 2012, 45, 2211-2221.

7 (a) M. Kathan, F. Eisenreich, C. Jurissek, A. Dallmann, J. Gurke and S. Hecht, Nat. Chem., 2018, 10, 1031-1036; (b) L. A. Ingerman and M. L. Waters, J. Org. Chem., 2009, 74, 111-117; (c) S. Wang, L. Yue, Z.-Y. Li, J. Zhang, H. Tian and I. Willner, Angew. Chem. Int. Ed., 2018, 57, 8105-8109.

8 F. Ulatowski, K. Dabrowa and J. Jurczak, Tet. Lett., 2016, 57, 1820-1824.

9 D. Larsen, S. R. Beeren, Chem. Sci. 2019, DOI: 10.1039/c9sc03983j.

10 (a) Y. B. Tewari, R. N. Goldberg and M. Sato, Carbohydr. Res., 1997, 301, 11-22; (b) J. C. M. Uitdehaag, B. A. van der Veen, L. Dijkhuizen and B. W. Dijkstra, Enzyme Microb. Technol., 2002, 30, 295-304; (c) J. C. Uitdehaag, K. H. Kalk, B. A. van der Veen, L. Dijkhuizen and B. W. Dijkstra, J. Biol. Chem., 1999, 274, 34868-34876.

11 (a) M. Zhu and H. Zhou, Org. Biomol. Chem., 2018, 16, 84348445. (b) A. A. Beharry and G. A. Wooley, Chem. Soc. Rev., 2011, 40, 4422-4437.

12 S. Crespi, N. A. Simeth and B. König, Nat. Chem. Rev., 2019, 3, 133-146.

13 D. Wang, W. Zhai, Q. Wei, C. Zhao and Y. Zheng, ChemPhotoChem, 2018, 2, 403-415.

14 S. Meier and S. R. Beeren, J. Am. Chem. Soc., 2014, 136, 1128411287.

15 (a) P. T. Corbett, S. Otto and J. K. M. Sanders, Chem. Eur. J., 2004, 10, 3139-3143; (b) P. T. Corbett, J. K. M. Sanders and S. Otto, J. Am. Chem. Soc., 2005, 127, 9390-9392.

16 M. Kathan, S. Hecht, Chem. Soc. Rev., 2017, 46, 5536-5550. 